\title{
A Review of the State of Art in Applying Biot Theory to Acoustic Propagation through the Bone
}

\author{
Haydar Aygün1*, Keith Attenborough2, Michiel Postema ${ }^{3}$ \\ ${ }^{1}$ Maritime and Technology Faculty, East Park Terrace, Southampton Solent University, Southampton, UK \\ ${ }^{2}$ Department of Engineering and Innovation, The Open University, Milton Keynes, UK \\ ${ }^{3}$ Department of Physics and Technology, University of Bergen, Bergen, Norway \\ Email: " haydar.aygun@solent.ac.uk, keith.attenborough@open.ac.uk, Michiel.Postema@ift.uib.no
}

Received 18 October 2014; revised 25 November 2014; accepted 11 December 2014

Copyright (C) 2014 by author and OALib.

This work is licensed under the Creative Commons Attribution International License (CC BY).

http://creativecommons.org/licenses/by/4.0/

(c) (i) Open Access

\begin{abstract}
Understanding the propagation of acoustic waves through a liquid-perfused porous solid framework such as cancellous bone is an important pre-requisite to improve the diagnosis of osteoporosis by ultrasound. In order to elucidate the propagation dependence upon the material and structural properties of cancellous bone, several theoretical models have been considered to date, with Biot-based models demonstrating the greatest potential. This paper describes the fundamental basis of these models and reviews their performance.
\end{abstract}

Keywords

Acoustic, Propagation, Bone, Theoretical Model

Subject Areas: Applied Physics, Theoretical Physics

\section{Introduction}

Understanding the propagation of acoustic waves through cancellous bone is an important pre-requisite to improve the diagnosis of osteoporosis by ultrasound. Bone essentially has two types of structure, both having the same mineralised collagen composition. Cortical bone has porosity less than $30 \%$ and may generally be considered to be solid; cancellous bone has porosity greater than $30 \%$ and consists of a complex open-celled porous network of rod- and plate-shaped elements termed trabeculae. The porosity of human cancellous bone ranges between $70 \%$ and $95 \%$, the remaining volume being perfused with bone marrow. In the adult human vertebral body for example, both horizontal and vertical trabeculae range from $50-120 \mu \mathrm{m}$ in thickness and space at in-

* Corresponding author.

How to cite this paper: Aygün, H., Attenborough, K. and Postema, M. (2014) A Review of the State of Art in Applying Biot Theory to Acoustic Propagation through the Bone. Open Access Library Journal, 1: e994.

http://dx.doi.org/10.4236/oalib.1100994 
tervals of between $1200-5000 \mu \mathrm{m}$ and 700 and $2000 \mu \mathrm{m}$ respectively [1].

Osteoporosis leads to nearly 9 million fractures annually worldwide [2], and over 300,000 patients present with fragility fractures to hospitals in the UK each year [3]. Direct medical costs from fragility fractures to the UK healthcare economy were estimated at $£ 1.8$ billion in 2000, with the potential to increase to $£ 2.2$ billion by 2025, and with most of these costs relating to hip fracture care [4].

Two mechanisms give rise to the structure of bone, modelling and remodelling. "Modelling" is the process primarily responsible for maintaining bones in their correct shape as they grow and respond to its biomechanical environment; it also controls the cortical thickness and marrow cavity diameter of bones as they age. "Remodelling" is mainly concerned with the continual replacing of old cancellous bone and occurs at discrete foci on the surface of the trabeculae. During remodelling, osteoclast cells create a resorption cavity that is subsequently filled with new collagen by osteoblast cells. In osteoporosis, there is an asymptomatic negative imbalance in remodelling, thereby creating a bone loss, particularly at sites of predominantly cancellous bone such as the spine, hip, wrist, and heel; this ultimately leads to skeletal fragility and increased risk of fracture [5].

The conventional method of assessing osteoporosis in the clinical environment is bone mineral density (BMD, $\mathrm{g} \cdot \mathrm{cm}^{-2}$ ), an areal parameter describing the bone mineral content (BMC) within a projected area. BMD is generally measured at sites most at risk of osteoporotic fracture, the spine, hip and wrist. BMD is generally measured using the technique of dual energy X-ray absorptiometry (DXA) [6]. The body may be considered to consist of three tissue components: bone, lean and fat. By assuming a certain proportion of lean/fat tissue overlying a bone, the BMC may be derived by solving simultaneous attenuation equations for two X-ray energies. True volumetric bone density may be derived using quantitative computed tomography (QCT) utilising a conventional CT scanner, a calibration phantom being scanned with the subject to convert Hounsfield numbers into $\mathrm{g} \cdot \mathrm{cm}^{-3}$ [7]. QCT is increasingly being used, particularly at the lumbar spine although there is a higher radiation dose compared to DXA. Although generally utilised as a research tool for the measurement of excised tissue samples obtained from the pelvis, micro CT provides a typical spatial resolution of $0.01 \mathrm{~mm}$ and hence replicates the true trabecular structure, compared to resolutions of approximately $1 \mathrm{~mm}$ for both DXA and QCT. A technique that is gaining increasing interest is magnetic resonance imaging (MRI) which essentially measures the water content of tissues. Bone does not therefore give an MR signal, although its presence may be inferred from a "negative" image [8].

Quantitative Ultrasound (QUS) generally involves measurements of the transmission of ultrasonic signals, either along a cortical bone surface or through a bone such as the heel and phalanx [9]. There are two fundamental measurement parameters, velocity $\left(\mathrm{ms}^{-1}\right)$ and attenuation $(\mathrm{dB})$. Velocity is obtained by dividing the propagation distance by the corresponding transit time, with through-transmission measurements recorded at the calcaneus (heel) and phalanx, and surface-transmission recorded primarily at the tibia. Attenuation is generally expressed as Broadband Ultrasound Attenuation (BUA, $\mathrm{dB} \cdot \mathrm{MHz}^{-1}$ ) at the calcaneus, describing the linear increase in attenuation with frequency between $200 \mathrm{kHz}$ and $600 \mathrm{kHz}$. It has been clinically demonstrated that velocity provides higher precision, expressed as CV\% whereas BUA exhibits higher dynamic range. It is generally accepted that of the QUS options, BUA measurement at the calcaneus provides the most accurate indication of osteoporotic fracture risk, particularly for hip fracture. Due to technical limitations, ultrasound measurements cannot be performed routinely at the common anatomical sites affected by osteoporosis (spine, hip and wrist).

A fundamental relationship exists linking the velocity $(v)$ of a sound wave to the elasticity $(E)$ and density $(\rho)$ of a material, namely $v=\sqrt{ }(E / \rho)$ although the elasticity modulus used in the relationship is dependent upon the sound propagation mode; for example Bulk modulus $+4 / 3$ of the Rigidity modulus for the longitudinal wave (), the rigidity modulus for the shear wave and the Young's modulus for the bar wave. Young's modulus is derived from mechanical testing and longitudinal velocity from ultrasound measurements [10]. A similar fundamental relationship does not exist for BUA. It has been shown however that BUA follows a parabolic-type dependence on porosity having a minimum values corresponding to both solid bone ( $0 \%$ porosity) and marrow (100\% porosity) [11]. Hence, similar BUA values may be obtained for a few marrow pores within a largely solid bone and a few bony trabeculae within a largely marrow sample. A parameter that follows a similar pattern is the surface area of the bone-marrow interface; associated with this, a linear relationship between BUA and fractal dimension has been demonstrated [12].

Even though more than twenty years have passed since BUA was first described [13], there remains a lack of a fundamental understanding of the dependence of ultrasound propagation, and BUA, in particular, upon the material and structural properties of cancellous bone. In order to elucidate these relationships, a number of theo- 
retical approaches have been considered including scattering, simple mixtures, idealized microstructures, and Biot. Scattering is caused by sudden spatial changes in elastic properties, the magnitude being dependent on relative size of in-homogeneities and the ultrasound wavelength. Multiple Scattering may also be considered, being a combination of the original and previously scattered waves. Boutin [14] has combined rigid-porous theory and scattering to investigate the low frequency scattering of acoustic wave propagation in heterogeneous media made of air and motionless inclusions. It should be noted however that scattering theories are only valid for low concentrations of isolated inhomogeneity and are not generally applicable to the study of cancellous bone. The simple mixture theory expresses velocity in terms of bone volume fraction, density, and bulk modulus. Chernov's theory [15] combines scattering and simple mixture theory via velocity fluctuations and scatterer size. Simple mixture theories have also had limited success for porous media such as cancellous bone. Two theories that are inherently applicable to the solid framework perfused with a visco-elastic fluid are the Schoenberg and Biot theories. Schoenberg's theory [16]-[18] considers periodically alternating parallel solid-fluid layers but does not consider the viscous absorption. The theory predicts two compressional waves, often referred to as "fast" and "slow" respectively, when the waves propagating through the solid frame of bone and marrow are inphase and out-of-phase respectively. The angular dependences of phase velocities for the fast and the slow waves in cancellous bone have been predicted [19], along with the anisotropic behaviour of acoustic wave propagation [20]. Biot theory was specifically developed to describe acoustic wave propagation in fluid-saturated porous elastic media [21] [22]; although originating for geophysical testing of porous rocks, it has been used extensively to describe the wave motion in cancellous bone. The Biot theory allows for an arbitrary microstructure, with separate motions considered for the solid elastic framework (bone) and the interspersed fluid (marrow), induced by the ultrasonic wave, and also includes energy loss due to viscous friction between solid (bone) and fluid (marrow). In addition to the two compressional waves predicted by Schoenberg's theory, the Biot theory also predicts a shear wave. McKelvie and Palmer [23] were first to apply Biot theory to ultrasonic wave propagation in cancellous bone. Hosokawa and Otani [24] first observed experimentally the two theoretically predicted compressional waves in cancellous bone at ultrasonic frequencies. The Biot model has since been used extensively to describe the wave motion in trabecular (cancellous) bone [25]-[28]. Attenborough et al. [29] presented tortuosities deduced from audio-frequency measurements in air-filled cancellous bone replicas and showed that there was strong anisotropy. The Biot theory has been further developed including semi-analytical approach that allows for transverse anisotropy in the frame elastic moduli, tortuosity and permeability for geophysical applications [30]. A modified Biot-Attenborough (MBA) model has also been proposed for acoustic wave propagation in a non-rigid porous medium with circular cylindrical pores starting from a formulation for a rigid-framed porous material [31]-[33]. The MBA has been used to predict the dependences of velocity and attenuation on frequency and porosity in bovine cancellous bone [34] [35]. The Biot model has also been modified to include the acoustic anisotropy of cancellous bone by introducing empirical angle-dependent parameters, and used to predict both the fast and slow wave velocities as a function of propagation angle with respect to the trabecular alignment of cancellous bone [36].

Previous work on the influence of anisotropic pore structure and elasticity in cancellous bone has been extended by developing an anisotropic Biot-Allard model allowing for angle dependent tortuosity and elasticity by Aygün et al. [37]. The extreme angle dependence of tortuosity corresponding to the parallel plate microstructure used by Hughes et al. [20] has been replaced by angle dependent tortuosity values based on data for slow wave transmission through air-filled bone replicas. Audio-frequency data obtained at audio-frequencies in air-filled bone replicas are used to derive an empirical expression for the angle-and porosity-dependence of tortuosity.

Most recently, Aygün et al. [38] [39] have transmitted ultrasonic signals through water saturated stereolithograpical bone replicas and through rigid porous ceramics [40]. Predictions of a modified anisotropic Biot-Allard model, which neglects scattering have been compared to measurements made at normal and oblique incidence in a water filled tank at $100 \mathrm{kHz}$ and $1 \mathrm{MHz}$. Remarkably, it is found that the expected occurrence of scattering does not cause significant discrepancies between predictions and data at $100 \mathrm{kHz}$ (which would be equivalent to $1.3 \mathrm{MHz}$ in real bone), perhaps as a consequence of the fact that the samples behave as low pass filters. Scattering should be even more important at $1 \mathrm{MHz}$ (equivalent to $13 \mathrm{MHz}$ in real bone) where the fast and slow wavelengths are $3 \mathrm{~mm}$ and $1.5 \mathrm{~mm}$ respectively. Nevertheless the modified Biot-Allard theory is found to predict the observed simple relationship between incident and transmitted waveforms at $1 \mathrm{MHz}$. Another effect of the structural anisotropy will be variation of permeability with direction. 


\section{The Biot Model}

The Biot theory predicts the complex velocities of two compressional waves and a single shear wave, given by;

$$
\begin{gathered}
V_{\text {fast,slow }}^{2}=\frac{\Delta \pm\left[\Delta^{2}-4\left(P R-Q^{2}\right)\left(\rho_{11} \rho_{22}-\rho_{12}^{2}\right)\right]^{2}}{2\left(\rho_{11} \rho_{22}-\rho_{12}^{2}\right)} \\
V_{\text {shear }}^{2}=\frac{N}{(1-\phi) \rho_{s}+(1-1 / \alpha) \rho_{f}}
\end{gathered}
$$

where $\Delta=P \rho_{22}+R \rho_{11}-2 Q \rho_{12}, P Q R$ are generalized elastic constants, $\rho_{11}, \rho_{22}$, and $\rho_{12}$ are mass coefficients which take into account the fact that the relative fluid flow through the pores is not uniform, $\rho_{s}$ is the mass density of the solid, $\rho_{f}$ is the mass density of the fluid, and $\alpha$ is the tortuosity of the medium. The elastic constants are given by;

$$
\begin{gathered}
P=\frac{(1-\phi)\left(1-\phi-\frac{K_{b}}{K_{s}}\right) K_{s}+\phi \frac{K_{s}}{K_{f}} K_{b}}{1-\phi+\phi \frac{K_{s}}{K_{f}}-\frac{K_{b}}{K_{s}}}+\frac{4 N}{3} \\
Q=\frac{\left(1-\phi-\frac{K_{b}}{K_{s}}\right) \phi K_{s}}{1-\phi+\phi \frac{K_{s}}{K_{f}}-\frac{K_{b}}{K_{s}}} \\
R=\frac{\phi^{2} K_{s}}{1-\phi+\phi \frac{K_{s}}{K_{f}}-\frac{K_{b}}{K_{s}}}
\end{gathered}
$$

where $K_{b}, K_{f}$, and $K_{s}$ are the bulk module of skeletal frame, fluid, and solid, respectively; $N$ is the shear modulus and $\phi$ is the porosity.

The bulk modulus of the skeletal frame $\left(K_{b}\right)$ and the bulk modulus of the solid $\left(K_{s}\right)$ are given by;

$$
K_{b}=\frac{E_{b}}{3\left(1-2 v_{b}\right)}, \quad K_{s}=\frac{E_{s}}{3\left(1-2 v_{s}\right)}
$$

where $v_{b}$ and $v_{s}$ are the Poisson's ratio of the skeletal frame and the solid, respectively, $E_{b}$ is the Young's modulus of the skeletal frame given by $E_{b}=E_{s}(1-\phi)^{n}$, where $E_{s}$ is the Young's modulus of the solid, and $n$ depends on the alignment of the structure [41].

The total mass of the fluid-solid aggregate per unit volume is given by $\rho=\rho_{11}+2 \rho_{12}+\rho_{22}$. The coefficient $\rho_{12}$ represents a mass coupling parameter between fluid and solid, $\rho_{11}$ represents the total effective mass of the solid moving in the fluid, and $\rho_{22}$ depends on the geometry of the frame not on the viscosity of the fluid [21]. The mass coefficients may be written

$$
\begin{gathered}
\rho_{11}=\rho_{1}+\rho_{a} \\
\rho_{22}=\rho_{2}+\rho_{a} \\
\rho_{12}=-\rho_{f} \phi(\alpha-1)
\end{gathered}
$$

where $\rho_{a}$ is the inertial coupling term given by $\rho_{a}=-\rho_{12}, \rho_{1}$ is the mass of solid per unit volume of aggregate given by $\rho_{1}=\rho_{s}(1-\phi)$, and $\rho_{2}$ is the mass of fluid per unit volume of aggregate given by $\rho_{2}=\phi \rho_{f}$.

Johnson et al. [42] have presented the dynamic tortuosity as;

$$
\alpha(\omega)=\alpha_{\infty}\left(1+\frac{\eta \phi}{j \omega \alpha_{\infty} \rho_{f} k_{0}} \sqrt{1+j \frac{4 \alpha_{\infty}^{2} k_{0}^{2} \rho_{f} \omega}{\eta \Lambda^{2} \phi^{2}}}\right)
$$


where $\alpha_{\infty}$ is the tortuosity, $\Lambda$ is the viscous characteristic length, $\eta$ is the viscosity, and $k_{0}$ is the permeability.

Biot theory considers the material to be isotropic, although tortuosities deduced from audio-frequency measurements in air-filled bone replicas, assuming rigid-porous behaviour, have shown a strong anisotropy Hughes et al. [19] have stated that there has been a consistent discrepancy between measured and predicted attenuation. It should be noted also that Biot theory does not include thermal effects. When the saturating fluid is air rather than a liquid, thermal effects become important and a further modification of the Biot theory is necessary.

\section{The Biot-Attenborough Model}

The Modified Biot-Attenborough (MBA) model was developed to consider acoustic wave propagation in a nonrigid porous medium with circular cylindrical pores [31]. The formulation starts from Attenborough's model [32] which is concerned with rigid-framed media and, therefore, does not include the fast wave of the Biot theory, and treats the viscous and thermal effects in a separate manner. A non-rigid structure is then allowed for by introducing an additional parameter and boundary condition. A similar formulation to the MBA model has been employed [35] with three new phenomenological parameters, namely: the boundary condition, phase velocity, and impedance parameters. An idealised microstructure was assumed consisting of circular cylindrical pores and considered one-dimensional sound propagation along with the axes of the cylinders.

The continuity equation of one-dimensional acoustic wave propagation through a single circular cylindrical pore filled with fluid is given by;

$$
-\rho_{f} \frac{\partial\langle v\rangle}{\partial x}=\frac{\partial \rho}{\partial t}
$$

where $\langle v\rangle$ is the averaged particle velocity over the pore cross section for propagation in the $x$ direction, which is taken to be the direction normal to the surface of the model porous medium. The equation of motion is given by;

$$
\frac{\partial p}{\partial x} \rho_{c}(\omega) \frac{\partial\langle v\rangle}{\partial t}
$$

where $p$ is the acoustic pressure, $\omega$ is the angular frequency, and $\rho_{c}(\omega)$ is the frequency-dependent density given by

$$
\rho_{c}(\omega)=\rho_{f}\left[1-2(\lambda \sqrt{i})^{-1} T(\lambda \sqrt{i})\right]^{-1}
$$

where

$$
T(\lambda \sqrt{i})=\frac{J_{1}(\lambda \sqrt{i})}{J_{0}(\lambda \sqrt{i})}
$$

$i=\sqrt{-1}, \quad J_{0}$ and $J_{1}$ are the cylindrical Bessel functions of the zeroth and the first order, respectively, and $\lambda$ is the dimensionless parameter related to the thickness of the viscous boundary layer at a pore wall and given by $\lambda=a m_{1}(\omega / v)^{1 / 2}$ where $a$ is the radius of the circular cylindrical pore, $m_{1}$ is a boundary condition parameter allowing for non-rigidity of the pore frame, and $v$ is the kinematic viscosity of the fluid.

The frequency-dependent compressibility of the fluid within a pore of arbitrary (but constant) cross section is given by;

$$
C_{c}(\omega)=\left(\frac{1}{\rho_{f}}\right)\left(\frac{\mathrm{d} \rho_{c}}{\mathrm{~d} p}\right)=\left(\rho_{f} c_{f}^{2}\right)^{-1}\left[1+2(\gamma-1) x\left(N_{\mathrm{Pr}}^{1 / 2} \lambda \sqrt{i}\right)^{-1} T\left(N_{\mathrm{Pr}}^{1 / 2} \lambda \sqrt{i}\right)\right]
$$

where $\gamma$ is the specific heat ratio of the fluid, $N_{\mathrm{Pr}}$ is the Prandtl number, and $c_{f}$ is the equilibrium sound velocity of the fluid.

When the analysis, based on the sound propagation through a single circular cylindrical pore, is extended to a bulk non-rigid porous medium, the complex propagation constants of the fast and slow waves are given, respec- 
tively, by:

$$
\begin{aligned}
& k_{\text {fast }}=\alpha_{\infty} k_{s} \sqrt{\frac{1}{(1-\phi)^{m_{2}}+\phi^{m_{2}}\left(k_{s} / k_{c}\right)^{2}}} \\
& k_{\text {slow }}=\alpha_{\infty} k_{g} \sqrt{\frac{1}{(1-\phi)^{m_{2}}+\phi^{m_{2}}\left(k_{g} / k_{c}\right)^{2}}},
\end{aligned}
$$

where $k_{s}$ is the propagation constant of the pore frame given by $k_{s}=\omega / c_{s}$ where $c_{s}$ is the pore frame velocity, $k_{c}$ is the propagation constant of the fluid in the pore given by $k_{c}=\omega C_{c}(\omega) \rho_{c}(\omega), k_{s}$ is the wave number of a hypothetical fluid with an extremely low wave velocity, and $m_{2}$ is a phase velocity fitting parameter.

The phase velocities and attenuation coefficients of the fast and slow waves can be obtained from the real and imaginary part of the complex propagation constant of the fast and slow waves respectively.

\section{The Schoenberg Model}

The Schoenberg model considers periodically alternating parallel solid-fluid layers, assuming that the viscous skin depth is much less than the fluid layer thickness when the fluid layer is ideal. Schoenberg has expressed acoustic wave propagation in terms of slowness vector given by $s=\left(s_{1}, s_{2}, s_{3}\right)$. The components of the slowness vector parallel to the layers, $s_{1}$, and normal to the layers, $s_{3}$, are related by;

$$
\frac{s_{3}^{2}}{\langle\rho\rangle}-\left[\frac{\phi\left(V_{f}^{-2}-s_{1}^{2}\right)}{\rho_{f}}+\frac{(1-\phi)\left(V_{f}^{-2}-s_{1}^{2}\right)}{\rho_{s}\left(1-V_{p l}^{2} s_{1}^{2}\right)}\right]=0
$$

where $V_{f}$ is the sound speed of the fluid, $V_{s}$ is the compressional speed of the solid, $\langle\rho\rangle$ is given by $\langle\rho\rangle=\phi \rho_{f}+(1-\phi) \rho_{s}, V_{p l}$ is the plate velocity given by;

$$
V_{p l}^{2}=4 V_{s h}^{2}\left(1-\frac{V_{s h}^{2}}{s^{2}}\right)
$$

where $V_{\text {sh }}$ is the shear speed of the solid.

The phase velocity can be found from the inverse of the magnitude of the vector, $\left(|s|^{-1}=1 /\left(s_{1}^{2}+s_{3}^{2}\right)^{1 / 2}\right)$ and the propagation angle with respect to the layering $\left(\theta=\tan ^{-1}\left(s_{3} / s_{1}\right)\right)$. Schoenberg's theory predicts two compressional waves equivalent to the acoustic waves propagating through the solid frame and fluid being in-phase and out-of-phase respectively. Inertial coupling varies with propagation angle relative to the stratification. For propagation parallel to the layers, inertial coupling is zero, and waves may propagate in the fluid and solid independently [19].

\section{The Anisotropic Biot-Hughes Model}

The Anisotropic Biot model incorporates viscous effects and anisotropic effects into the Biot model in a simple and straightforward manner [20], assuming that the degree of inertial coupling within Schoenberg's layers are equivalent to that occurring in an arbitrary anisotropic porous medium as described by the Biot's theory. An angle dependent tortuosity was introduced to describe a layered structure in the Biot's theory, from which the angle dependant tortuosity may be obtained by equating the compressional phase velocity in terms of $\alpha$ from the Biot's theory [Equation (1)], with that from Schoenberg's model in terms of propagation angle, $\theta$, [Equations (18) and (19)]. Shear in the solid is neglected.

( $K_{b}=N=0$ and $P R-Q^{2}=0$ ). The tortuosity was found from

$$
\alpha(\theta)=1+\left[\frac{(1-\phi) \rho_{s}}{\langle\rho\rangle}\right] \cot ^{2} \theta
$$


The angle-dependent tortuosity given by Equation (20) should be substituted for $\alpha_{\infty}$ to predict wave properties that change with orientation. Hughes et al. have observed a significant discrepancy, for predictions of the dependence of the fast wave speed on angle, between the Stratified Biot model and Schoenberg's theory, disagreeing by almost $50 \%$ at $0^{\circ}$. They have also stated that the Stratified Biot model gives poorer agreement with slow wave data than the Schoenberg model, both quantitatively and in the curvature of its angular variation.

\section{The Anisotropic Biot-Attenborough Model}

Aygün et al. [37] have combined the heuristic form of angle-dependent elasticity suggested by Lee et al. [36] in Biot-Allard theory with a heuristic angle and porosity dependent tortuosity function based on data obtained at audio frequencies with air-filled (human) bone replicas by Attenborough et al. [29]. The heuristic form for porosity and angle dependent tortuosity may be written:

$$
\alpha_{\infty}=1-r\left(1-\frac{1}{\phi}\right)+k \cos ^{2}(\theta)
$$

where $r$ and $k$ can be considered adjustable.

The assumed angle dependence function is chosen arbitrarily but is simple and consistent with the expected variation in fast wave speed with angle. A range of possible values of $r$ and $k$ have been found by comparing predictions of Equation (21) for $\theta=0^{\circ}$ and $90^{\circ}$ respectively with values deduced from air-filled replica bones (Attenborough et al. [29]) of known porosity. Values of $r$ and $k$ are found by solving the resulting simultaneous equations. The value of $r$ is predicted to have important influence on the fast wave speed variation with porosity perpendicular to the dominant structural orientation and on the slow wave speed variation with porosity parallel to the dominant structural orientation.

Aygün et al. [38] have reported measurements of ultrasonic transmission made through water-saturated bone replicas at $100 \mathrm{kHz}$ and $1 \mathrm{MHz}$. The resulting data are compared with predictions of a modified Biot-Allard model with anisotropic angle-and-porosity dependent tortuosity, and angle-dependent elasticity. Transmitted signals for water saturated stereolithograpical bone replicas have been predicted by modified anisotropic BiotAllard model, which neglects scattering, and the results have been compared to measurements made in a water filled tank at $100 \mathrm{kHz}$ and $1 \mathrm{MHz}$. The wavelengths of the slow and fast wave in water-saturated STL bone replicas at $100 \mathrm{kHz}$ are $15 \mathrm{~mm}$ and $30 \mathrm{~mm}$, respectively. These wavelengths are comparable with the dimensions of microstructural elements of STL bone replicas. According to Williams [43], the pore sizes in cancellous bone vary between 0.5 and $1 \mathrm{~mm}$, so typical trabeculae widths in the replicas vary between 6.5 and $13 \mathrm{~mm}$. Remarkably, scattering seems not to cause significant discrepancies between predictions and data at $100 \mathrm{kHz}$ (which would be equivalent to $1.3 \mathrm{MHz}$ in real bone), perhaps as a consequence of the fact that the samples behave as low pass filters. Scattering should be more important at $1 \mathrm{MHz}$ (equivalent to $13 \mathrm{MHz}$ in real bone) where the fast and slow wavelengths are $3 \mathrm{~mm}$ and $1.5 \mathrm{~mm}$ respectively. So the agreement between predictions and data is rather surprising. These data and predictions support further use of Biot-based theories and of STL replicas for studying ultrasonic transmission through bone.

Aygün et al. [39] have investigated further ultrasonic wave transmission in water-saturated bone replicas at 1 $\mathrm{MHz}$ as a function of angle. The predictions of the anisotropic Biot-Allard model allowing for angle-dependent elasticity and angle-and-porosity dependent tortuosity have been compared with measurements of pulses centered on $1 \mathrm{MHz}$ transmitted through water saturated stereo-lithographical (STL) bone replicas which are thirteen times larger than the original bone samples at normal and oblique angles. The predictions and data are in reasonable agreement despite the expected role of scattering.

\section{Discussion}

Although Schoenberg's theory predicts two compressional waves equivalent to the wave of the first kind and the wave of the second kind of Biot's theory, it does not predict the shear wave of the Biot's theory. Schoenberg's theory does not consider the fluid viscosity, and thermal effects, and consequently, it is not possible to use Schoenberg's theory to predict wave attenuation or to make comparisons with the absorption predicted by Biot theory. The anisotropic behaviour of acoustic wave propagation in cancellous bone using the Schoenberg theory has been investigated, Hughes et al. [19] stating that the inclusion of viscosity in the Biot's theory gives no sig- 
nificant advantage in respect of agreement with experimental findings, and that the omission of the viscosity in Schoenberg's model prevents it from accounting for viscous absorption and confines its application to Biot's "high" frequency region (Hughes et al. [20]).

Both the velocity and attenuation of sound as predicted by the Biot theory are dependent on frequency, the elastic properties of the constituent materials, porosity, permeability, tortuosity, and effective stress. The original Biot formulation introduced a frequency-independent pore shape parameter. In a subsequent refinement, the frequency-dependent viscous effects due to relative motion of fluid and solid in the porous medium are predicted to depend on a viscous characteristic length (Johnson et al. [42]). This is dominated by the smallest pore cross sections and is difficult to measure. When the saturating fluid is air rather than a liquid, thermal effects become important and a further modification of the Biot theory is necessary since the original formulation does not include them. Such a modification, which has been used mainly when modelling the acoustical properties of air-filled porous materials, used in building acoustics and engineering acoustics, introduces a thermal characteristic length (Allard [44]). Twice the inverse of thermal characteristic length represents the pore surface area per unit volume. In materials that are not affected by water saturation, this can be measured by a water suction method (Leclaire et al. [45]). For many materials the thermal characteristic length is approximately twice the viscous characteristic length. The inclusion of the thermal characteristic length is relevant to comparison of predictions with high frequency data obtained from air-saturated bone replicas. During acoustic excitation (e.g. from a loudspeaker) of an air-filled porous solid most of the acoustic energy travels in the pores and the acoustical properties are described well by assuming that the solid frame is rigid. On the other hand intimate mechanical contact between a transducer and a porous elastic medium excites waves predominantly in the solid frame. Fellah et al. [26] have presented an analytical model of the reflection and transmission coefficient of a slab of cancellous bone with an elastic frame based on the Biot theory modified by Johnson et al. [42] to describe the viscous interaction between fluid and structure. By comparing predictions with laboratory data on ultrasonic pulse transmission through water-filled samples of human cancellous bone, Fellah et al. [26] have concluded that the modified Biot theory using Johnson et al. [42] model is suitable for describing the propagation of ultrasonic wave in cancellous bone. A significant attraction of the Biot theory is that it includes structurally dependent parameters including permeability and tortuosity as well as the elastic constants of the porous frame and frame material (Allard [44]). An important limitation of the Biot theory is that it requires that the acoustic wavelengths to be large compared with the microstructural features (such pores and trabeculae in cancellous bone). Another difficulty with the Biot theory is that it requires knowledge of many parameters, some of which are hard to determine.

The inherent anisotropy of cancellous bone means that the acoustical properties vary with transmission direction. Tortuosities deduced from audio-frequency measurements in air-filled bone replicas, assuming rigid-porous behaviour, have shown a strong anisotropy [25]. To predict fast wave transmission it is necessary to allow for elastic anisotropy also. The trabeculae in cancellous bone suggest transverse anisotropy: an idealized version of which, for example, is parallel plates (Hughes et al. [19]). A method of including the effects of anisotropy in the Biot theory (Hughes et al. 2007) introduces an angle dependent tortuosity, based on the parallel plate idealization of the microstructure, and a heuristic angle dependent Young's modulus. However the approach of Hughes et al. [20] did not yield particularly good predictions of the angle dependence of fast wave speeds.

Lee et al. [36] have stated that the MBA model predicts a slightly negative dispersion of phase velocity linearly with frequency and the nonlinear relationships of attenuation and BUA with porosity. The experimental results presented by Lee et al. [36] are in good agreement with the theoretical results estimated with the MBA model.

The anisotropic pore structure and elasticity of cancellous bone cause wave speeds and attenuation in cancellous bone to vary with angle. Aygün et al. [37] have extended previous work on the influence of anisotropic pore structure and elasticity in cancellous bone by developing an anisotropic Biot-Allard model allowing for angle-dependent elasticity, and angle-and-porosity dependent tortuosity. The extreme angle dependence of tortuosity corresponding to the parallel plate microstructure used by Hughes et al. [20] has been replaced by angle-andporosity dependent tortuosity values based on data for slow wave transmission through air-filled stereolithography (STL) bone replicas Aygün et al. [38]. It has been suggested that the anisotropic Biot-Allard model could be used to give further insight into the factors that have the most important influence on the angle dependency of wave speeds and attenuation in cancellous bone. Nevertheless the applicability of Biot-based theories to ultrasonic propagation in bone remains in question given the expected role of scattering which is neglected in the 
these theories.

Predictions of a modified anisotropic Biot-Allard theory by Aygün et al. [39] have been compared with measurements of pulses centered on $1 \mathrm{MHz}$ transmitted through water saturated stereo-lithographical (STL) bone replicas which are thirteen times larger than the original bone samples at normal and oblique angles. The predictions and data are in reasonable agreement despite the expected role of scattering.

The likely ranges of validity for Biot modelling approaches can be discussed in terms of the ratio $(\varepsilon=l / L)$ of a characteristic inhomogeneity size $(l)$ and the reduced sound wavelength $L=\lambda / 2 \pi(\lambda)$. Scattering is likely to be significant for values of $\varepsilon$ greater than 1 whereas Biot theory was derived for values of $\varepsilon$ that are significantly less than 1 . At $1 \mathrm{MHz}$ the fast and slow wavelengths are $3 \mathrm{~mm}$ and $1.5 \mathrm{~mm}$ respectively. For the fast waves the values of the ratios, $\varepsilon$, in FRA and CAB replicas are 0.1257 and 0.3142 respectively, and for the slow waves the values are 0.2513 and 0.6283 . These indicate clearly that scattering should be significant so the agreement between Biot-based predictions and data at $1 \mathrm{MHz}$ is rather surprising.

\section{Conclusions and Further Work}

Several models being used for acoustic wave propagation in cancellous bone have been presented and reviewed. Of these, the various forms of Biot theory have shown some promise. That by Fellah et al. [26], has been found to give excellent agreement with data obtained from transmitting ultrasonic pulses through water-filled samples of human cancellous bone. Using another version of Biot theory, Lee et al. [36] have observed that the phase velocity is approximately non-dispersive and the attenuation coefficient linearly increases with frequency. Lee et al. [34] also state that the experimental results are in good agreement with the theoretical results estimated with the MBA model which predicts a slightly negative linear dispersion of phase velocity with frequency and the nonlinear relationships of attenuation and BUA with porosity. On the other hand, Haiat et al. [46] have stated that scattering effects are responsible for the negative values of dispersion whereas the frequency dependence of the attenuation coefficient in bone marrow and/or in the trabecular is shown to induce an increase in the dispersion. The existence of the trabeculae in cancellous bone means that it has transverse anisotropy. The inherent anisotropy of cancellous bone means that the acoustical properties vary with transmission direction [29]. To predict fast wave transmission it is necessary to allow for elastic anisotropy also. Although Hughes et al. [20] have presented a method of including the effects of anisotropy in Biot theory introducing an angle dependent tortuosity, based on the parallel plate idealization of the microstructure, and a heuristic angle dependent Young's modulus, their approach does not yield particularly good predictions of the angle dependence of fast wave speeds and another effect of the structural anisotropy will be variation of permeability with direction.

Aygün et al. [38] have stated that the use of stereolithograpical bone replicas made from resin has the potential to enable systematic investigations of the influences of perforation and thinning in cancellous bone on the acoustical and mechanical properties of the bone structure. Waves transmitted through STL bone replicas with higher porosity values have higher amplitudes. Osteoporotic bones will have higher porosity values due to bone loss, so greater energy will be transmitted through them in comparison with normal bone.

Another consequence of using replica bones which are 13 times the actual size of the bone microstructure is that scattering should become important at lower frequencies than that in measurements with real bone samples.

The predictions of the anisotropic Biot-Allard model allowing for angle-dependent elasticity and angle-andporosity dependent tortuosity have been compared with measurements made in a fluid (water) filled tank at 1 $\mathrm{MHz}$ by Aygün et al. [39]. It is found that predictions of the variation of transmitted waveforms with angle through two types of bone replica are in reasonable agreement with data despite the fact that scattering is not included in the theory.

A Finite Element Models (FEM), preferably an anisotropic Biot-based model modified by Aygün et al., [38]-[40], for cancellous bone should be developed. This would enable modelling of complete bone structures which would be more appropriate to the context of clinical monitoring. Such models of the acoustical properties of complex arrangements of anisotropic porous and elastic media are used increasingly in engineering studies, for example those related to the acoustical design of vehicle interiors [47]-[50].

Boutin [14] has discussed a general multiple-scale approach that allows for scattering and visco-thermal effects in a rigid-porous medium and has considered a specific application to a parallel plate medium. However, the development of model capable of covering both viscous and scattering regimes and applicable to an aniso- 
tropic poroelastic medium remains a formidable challenge.

\section{References}

[1] Thomsen, J.S., Ebbesen, E.N. and Mosekilde, L. (2002) Age-Related Differences between Thinning of Horizontal and Vertical Trabeculae in Human Lumbar Bone as Assessed by a New Computerized Method. Bone, 31, 136-142.

[2] Johnell, O. and Kanis, J.A. (2006) An Estimate of the Worldwide Prevalence and Disability Associated with Osteoporotic Fractures. Osteoporosis International, 17, 1726-1733. http://dx.doi.org/10.1007/s00198-006-0172-4

[3] British Orthopaedic Association (2007) The Care of Patients with Fragility Fracture.

[4] Burge, R.T., Worley, D. and Johansen, A. (2001) The Cost of Osteoporotic Fractures in the UK: Projections for 20002020. Journal of Medical Economics, 4, 51-52. http://dx.doi.org/10.3111/200104051062

[5] Rosen, C.J. (2004) Anatomy, Physiology and Disease. In: Langton, C.M. and Njeh, C.F., Eds. The Physical Measurement of Bone, IOPP, 3-34.

[6] Njeh, C.F. and Shepherd, J.A. (2004) Absorptiometric Measurement. In: Langton, C.M. and Njeh, C.F., Eds., The Physical Measurement of Bone, IOPP, 267-307.

[7] Lang, T. (2004) Quantitative Computed Tomography. In: Langton, C.M., \& Njeh, C.F. Eds., The Physical Measurement of Bone, IOPP, 308-318.

[8] Pothuaud, L. and Majumdar, S. (2004) Magnetic Resonance Imaging. In: Langton, C.M. and Njeh, C.F., Eds., The Physical Measurement of Bone, IOPP, 379-411.

[9] Njeh, C.F., Boivin, C.M. and Langton, C.M. (1997) The Role of Ultrasound in the Management of Osteoporosis: A Review. Osteoporosis International, 7, 7-22. http://dx.doi.org/10.1007/BF01623454

[10] Njeh, C.F., Hodgskinson, R., Currey, J.D. and Langton, C.M. (1996) Orthogonal Relationships between Ultrasonic Velocity and Material Properties of Bovine Cancellous Bone. Medical Engineering \& Physics, 18, 373-381. http://dx.doi.org/10.1016/1350-4533(95)00064-X

[11] Hodgskinson, R., Njeh, C.F., Whitehead, M.A. and Langton, C.M. (1996) The Non-Linear Relationship between BUA and Porosity in Cancellous Bone. Physics in Medicine \& Biology, 41, 2411-2420. http://dx.doi.org/10.1088/0031-9155/41/11/012

[12] Langton, C.M., Whitehead, M.A., Haire, T.J. and Hodgskinson, R. (1998) Fractal Dimension Predicts Broadband Ultrasound Attenuation in Stereolithography Models of Cancellous Bone. Physics in Medicine and Biology, 43, 467-471. http://dx.doi.org/10.1088/0031-9155/43/2/019

[13] Langton, C.M., Palmer, S.B. and Porter, R.W. (1984) The Measurement of Broadband Ultrasonic Attenuation in Cancellous Bone. Engineering in Medicine, 13, 89-91. http://dx.doi.org/10.1243/EMED_JOUR_1984_013_022_02

[14] Boutin, C. (2007) Rayleigh Scattering of Acoustic Waves in Rigid Porous Media. Journal of the Acoustical Society of America, 122, 1888-1905. http://dx.doi.org/10.1121/1.2756755

[15] Chernov, L. (1960) Wave Propagation in a Random Medium. McGraw-Hill, New York.

[16] Schoenberg, M. (1983) Wave Propagation in a Finely Laminated Periodic Elastoacoustic Medium. Applied Physics Letters, 42, 350-352. http://dx.doi.org/10.1063/1.93929

[17] Schoenberg, M. (1984) Wave Propagation in Alternating Solid and Fluid Layers. Wave Motion, 6, 303-320. http://dx.doi.org/10.1016/0165-2125(84)90033-7

[18] Schoenberg, M. and Sen, P.N. (1986) Wave Propagation in Alternating Solid and Viscous Fluid Layers: Size Effects in Attenuation and Dispersion of Fast and Slow Waves. Applied Physics Letters, 48, 1249-1251. http://dx.doi.org/10.1063/1.96994

[19] Hughes, E.R., Leighton, T.G., Petley, G.W. and White, P.R. (1999) Ultrasonic Propagation in Cancellous Bone: A New Stratified Model. Ultrasound in Medicine and Biology, 25, 811-821. http://dx.doi.org/10.1016/S0301-5629(99)00034-4

[20] Hughes, E.R., Leighton, T.G., White, P.R. and Petley, G.W. (2007) Investigation of an Anisotropic Tortuosity in a Biot Model of Ultrasonic Propagation in Cancellous Bone. Journal of the Acoustical Society of America, 121, 568-574. http://dx.doi.org/10.1121/1.2387132

[21] Biot, M.A. (1956) Theory of Propagation of Elastic Waves in a Fluid Saturated Porous Solid. I. Low Frequency Range. Journal of the Acoustical Society of America, 28, 168-178. http://dx.doi.org/10.1121/1.1908239

[22] Biot, M.A. (1956) Theory of Propagation of Elastic Waves in a Fluid Saturated Porous Solid. II. High Frequency Range. Journal of the Acoustical Society of America, 28, 179-191. http://dx.doi.org/10.1121/1.1908241

[23] McKelvie, M.L. and Palmer, S.B. (1991) The Interaction of Ultrasound with Cancellous Bone. Physics in Medicine 
and Biology, 36, 1331-1340. http://dx.doi.org/10.1088/0031-9155/36/10/003

[24] Hosokawa, A. and Otani, T. (1997) Ultrasonic Wave Propagation in Bovine Cancellous Bone. Journal of the Acoustical Society of America, 101, 558-562. http://dx.doi.org/10.1121/1.418118

[25] Haire, T.J. and Langton, C.M. (1999) Biot Theory: A Review of Its Application on Ultrasound Propagation through Cancellous Bone. Bone, 24, 291-295. http://dx.doi.org/10.1016/S8756-3282(99)00011-3

[26] Fellah, Z.E.A., Chapelon, J.Y., Berger, S., Lauriks, W. and Depollier, C. (2004) Ultrasonic Wave Propagation in Human Cancellous Bone: Application of Biot Theory. Journal of the Acoustical Society of America, 116, 61-73. http://dx.doi.org/10.1121/1.1755239

[27] Sebaa, N., Fellah, Z., Fellah, M., Ogam, E., Wirgin, A., Mitri, F., Depollier, C. and Lauriks, W. (2006) Ultrasonic Characterisation of Human Cancellous Bone Using the Biot Theory: Inverse Problem. Journal of the Acoustical Society of America, 120, 1816-1824. http://dx.doi.org/10.1121/1.2335420

[28] Pakula, M., Padilla, F., Laugier, P. and Kaczmarek, M. (2008) Application of Biot's Theory to Ultrasonic Characterization of Human Cancellous Bones. Journal of the Acoustical Society of America, 123, 2415-2423.

[29] Attenborough, K., Qin, Q., Fagan, M.J., Shin, H.C. and Langton, C.M. (2005) Measurements of Tortuosity in Stereolithographical Bone Replicas Using Audio-Frequency Pulses. Journal of the Acoustical Society of America, 118, 27792782.

[30] Carcione, J. (1996) Wave Propagation in Anisotropic, Saturated Porous Media: Plane-Wave Theory and Numerical Simulation. Journal of the Acoustical Society of America, 99, 2655-2666. http://dx.doi.org/10.1121/1.414809

[31] Roh, H.S., Lee, K.I. and Yoon, S.W. (2003) Acoustic Characteristics of a Non-Rigid Porous Medium with Circular Cylindrical Pores. Journal of the Korean Physical Society, 45, 55-62.

[32] Attenborough, K. (1982) Acoustical Characteristics of Porous Materials. Physics Reports, 82, 179-227. http://dx.doi.org/10.1016/0370-1573(82)90131-4

[33] Attenborough, K. (1983) Acoustic Characteristics of Rigid Fibrous Absorbents and Granular Materials. Journal of the Acoustical Society of America, 73, 785-799. http://dx.doi.org/10.1121/1.389045

[34] Lee, K.I., Roh, H.S. and Yoon, S.W. (2003) Acoustic Wave Propagation in Bovine Cancellous Bone: Application of the Modified Biot-Attenborough Model. Journal of the Acoustical Society of America, 114, 2284-2293. http://dx.doi.org/10.1121/1.1610450

[35] Lee, K.I. and Yoon, S.W. (2006) Comparison of Acoustic Characteristics Predicted by Biot's Theory and the Modified Biot-Attenborough Model in Cancellous Bone. Journal of Biomechanics, 39, 364-368. http://dx.doi.org/10.1016/j.jbiomech.2004.12.004

[36] Lee, K.I., Hughes, E.R., Humphery, V.F., Leighton, T.G. and Choi, M.J. (2007) Empirical Angle-Dependent Biot and MBA Models for Acoustic Anisotropy in Cancellous Bone. Physics in Medicine and Biology, 52, 59-73. http://dx.doi.org/10.1088/0031-9155/52/1/005

[37] Aygün, H., Attenborough, K., Postema, M., Lauriks, W. and Langton, C.M. (2009) Predictions of Angle-Dependent Tortuosity and Elasticity Effects on Sound Propagation in Cancellous Bone. Journal of the Acoustical Society of America, 126, 3286-3290. http://dx.doi.org/10.1121/1.3242358

[38] Aygün, H., Attenborough, K., Lauriks, W. and Langton, C.M. (2010) Ultrasonic Wave Propagation in Stereo-Lithographical Bone Replicas. Journal of the Acoustical Society of America, 127, 3781-3789. http://dx.doi.org/10.1121/1.3397581

[39] Aygün, H., Attenborough, K., Lauriks, W., Rubini, P.A. and Langton, C.M. (2011) Wave Propagation in StereoLithographical (STL) Bone Replicas at Oblique İncidence. Applied Acoustics, 72, 458-463. http://dx.doi.org/10.1016/j.apacoust.2011.01.010

[40] Aygün, H. and Barlow, C. (2015) Ultrasonic Wave Propagation in Porous Rigid Ceramics at Oblique Incidence. Applied Acoustics, 88, 6-11. http://dx.doi.org/10.1016/j.apacoust.2014.07.011

[41] Gibson, L.J. (1985) The Mechanical Behaviour of Cancellous Bone. Journal of Biomechanics, 18, 317-328. http://dx.doi.org/10.1016/0021-9290(85)90287-8

[42] Johnson, D.L., Koplik, J. and Dashen, R. (1987) Theory of Dynamic Permeability and Tortuosity in Fluid-Saturated Porous Media. Journal of Fluid Mechanics, 176, 379-402. http://dx.doi.org/10.1017/S0022112087000727

[43] Williams, J.L. (1992) Ultrasonic Wave Propagation in Cancellous and Cortical Bone: Predictions of Some Experimental Results by Biot's Theory. Journal of the Acoustical Society of America, 91, 1106-1112. http://dx.doi.org/10.1121/1.402637

[44] Allard, J.F. (1993) Propagation of Sound in Porous Media: Modelling Sound Absorbing Materials. Chapman and Hall, London.

[45] Leclaire, P., Swift, M.J. and Horoshenkov, K.V. (1998) Determining the Specific Area of Porous Acoustic Materials 
from Water Extraction Data. Journal of Applied Physics, 84, 6886-6890. http://dx.doi.org/10.1063/1.368985

[46] Haiat, G., Lhémery, A., Padilla, F., Laugier, P. and Naili, S. (2008) Modelling of “Anomalous” Velocity Dispersion in Trabecular Bone: Effect of Maultiple Scattering and of Viscous Absorption. Euro-Noise, Paris.

[47] Atalla, N., Panneton, R. and Deberque, P. (1997) A Mixed Displacement-Pressure Formulation for Poroelastic Materials. Journal of Acoustical Society of America, 104, 1444-1452. http://dx.doi.org/10.1121/1.424355

[48] Gorog, S., Panneton, R. and Atalla, N. (1997) Mixed Displacement-Pressure Formulation for Acoustic Anisotropic Open Porous Media. Journal of Applied Physics, 82, 4192-4196.

[49] Hörlin, N.E., Nordström, M. and Göransson, P. (2001) A 3-D Hierarchical FE Formulation of Biots Equations for Elastoacoustic Modeling of Porous Media. Journal of Sound and Vibration, 245, 633-652. http://dx.doi.org/10.1006/jsvi.2000.3556

[50] Panneton, R. and Atalla, N. (1998) An Efficient Scheme for Solving the Three-Dimensional Poroelasticity Problem in Acoustics. Journal of the Acoustical Society of America, 101, 3287-3298. http://dx.doi.org/10.1121/1.418345 